\title{
Simulations of Single Polymer Chains in the Dense Limit
}

\author{
Peter Grassberger and Rainer Hegger \\ Physics Department, University of Wuppertal D-5600 Wuppertal 1, FRG
}

November 12, 2017

\begin{abstract}
We present simulation results for single a-thermal chain polymers in finite volumes. For this we use a recently proposed recursive implementation of the enrichment method. In 3 dimensions it allows the simulation of extremely long chains (up to $N=300,000$ ). It is much less efficient for $d=2$, but we can also there extend considerably the previously accessible range of chain lengths and densities. We verify all tested scaling laws except one, and we point out similarities with complex optimization problems.
\end{abstract}

PACS numbers: $05.20,05.40,05.50,36.20 . \mathrm{E}, 61.40,64.70$ 


\section{Introduction}

Consider an ensemble of self avoiding chains ("SAW's") on a regular lattice in $d$ dimensions. All chains start from the origin of the lattice. Each configuration of a chain of length $N$ (here and in the following, $N$ will be the number of links, so that the number of sites touched by the chain is $N+1$ ) contributes to the partition function with a weight $z^{N}$, where $z$ is a constant ( $N$-independent) "fugacity". If we denote by $C_{N}$ the number of configurations with length $N$ and with one end fixed at the origin (with $C_{0}=1$ ), we have thus

$$
Z(z)=\sum_{N=0}^{\infty} z^{N} C_{N} .
$$

This provides a simple model for the grand canonical f partition sum of an isolated polymer in a good solvent, where the excluded volume interaction is taken into account by the requirement of self avoidance. Although details are obviously not very physical, it should give the correct scaling behavior in the limit of very long chains, $N \rightarrow \infty$.

It is thus not surprising that an enormous amount of effort - both analytical and numerical [1, 2] - has been devoted to the study of the above model. In particular it is known that the sum in eq.(1) converges up to a critical value $z_{c}=1 / \mu$, where $Z(z)$ has a singularity

$$
Z(z) \sim \frac{\text { const }}{(1-\mu z)^{\gamma}}
$$

Equivalently, $C_{N}$ obeys a scaling law $C_{N} \sim \operatorname{const} \mu^{N} N^{\gamma-1}$.

Most of the previous works have dealt with the subcritical phase $z<z_{c}$, and the supercritical case $z>z_{c}$ has obviously been studied much less. In order to define the theory for $z>z_{c}$, one has two alternatives: one can either make an analytic continuation, or one can regularize the sum in eq.(11) by a cut-off at large $N$. The latter can be done such that it has a very natural physical interpretation: we simply put the chain into a finite volume $V$, and count only those configurations which never leave the volume.

In the limit $V \rightarrow \infty$, we obtain thus a model for a single polymer chain which fills a macroscopic volume with finite monomer density $\rho$. In the literature this is known as the "dense limit" [3, 4]. In the following we shall study this model by means of Monte Carlo simulations.

Actually, the dense limit can also be understood as the limit of a more general model. There, the number of chains is fluctuating and controlled by a second fugacity $x$, and we take the limit $x \rightarrow 0$ [5]. There has been some controversy

\footnotetext{
${ }^{1}$ Notice that some authors [4] call this the equilibrium ensemble, in order to distinguish it from a truly grand canonical ensemble in which also the number of chains can fluctuate; in the present case, we always work with a single chain.
} 
in the literature following the claim by Gujurati [6] that there should be a phase transition in this model at a finite value of $x$. It seems now clear that this claim is wrong [4, 5], and we shall not mention this generalization further. In any case, the present paper will be devoted exclusively to the case $x=0$.

All relevant scaling laws at the transition from $\rho=0$ to $\rho>0$ can be obtained from the conventional (and well established) scaling theory for subcritical SAW's [1]. This will be reviewed in sec.3, where also Monte Carlo data are given in support of these scaling laws in 3 dimensions.

In the opposite limit, when $\rho=1$ such that the SAW visits every point of the lattice, we are dealing with hamiltonian walks. These have been studied in several papers, the most comprehensive study being that of [7]. They form a model for a dense polymer melt.

The intermediate region $0<\rho<1$ is particularly interesting in 2 dimensions, where it was shown in [3] that the model is a co-dimension 0 ("self-organized" [8]) critical phenomenon. That means that various observables are described by non-trivial scaling laws, although there is no need for a control parameter to take any special value. In 3 dimensions there should be no such scaling laws, but we expect logarithmic behavior instead.

It would of course be very desirable if one could verify these predictions numerically. Some numerical results based on transfer matrix calculations have been given in [3]. In that paper and in [13, 14], also Monte Carlo simulations were presented. But only very small systems could be simulated in [3], and systems with $>1$ chain (i.e., $x>0$ in the above sense) were simulated in 13, 14. Thus clear-cut and significant tests of the predictions of [3] would be most welcome. But all polymer Monte Carlo algorithms which we are aware of become inefficient at high densities.

It is the aim of the present paper to show that recursive implementations [9, 10] of the incomplete enumeration [11] and of the enrichment 12 methods can be quite efficient in simulating polymers in the dense limit.

This is so in spite of the problem that we cannot justify our algorithms rigorously, in the way we have to apply them in most dense limit problems. While this seems to be mainly a formal problem in $3 d$ (we do not encounter any serious problems unless we go to high densities), the situation is numerically very delicate in $2 d$. As a consequence we cannot go to really large systems and high densities, but we can go considerably much further than previous analyses. We will be able to check all scaling laws mentioned above with much higher accuracy than was possible previously. We will find good agreement in all cases except one.

The main problem in $2 d$ is that we cannot produce long chains if we from the beginning use a fugacity $z$ which is much larger than the critical one. A better strategy is to start building chains with $z$ very close to $z_{c}$, and to increase it during the growth of the chain. Finding the optimal schedule which gives the longest 
chains (i.e., the highest densities) is very similar to optimization in a problem with many local maxima, where greedy methods usually are sub-optimal.

In sec. 2 we shall present our algorithm and the problems encountered when applying it to dense systems. In sec. 3 we shall treat the limit of small densities, while our main results for scaling laws in $2 d$ systems will be discussed in sec.4. The very dense limit and hamiltonian walks are treated in sec.5. The paper concludes with a discussion in sec.6.

\section{Algorithm}

We used a recursive algorithm which can be considered as a recursive and randomized implementation of the old enrichment method [12, 10. In this algorithm we call a subroutine $\operatorname{STEP}(\mathbf{x}, N)$ in order to add the next $(N$-th) step to a chain starting at site $\mathbf{x}_{\mathbf{0}}=0$ and ending at $\mathbf{x}_{N}=\mathbf{x}$. Apart from updating whatever statistics we want to measure, this subroutine does the following: it first marks site $\mathbf{x}$ as occupied, calls itself a random number of times at one or more neighboring sites $\mathbf{x}^{\prime}=\mathbf{x} \pm \mathbf{e}_{i}$ and with $N$ replaced by $N+1$, and marks site $\mathbf{x}$ again as free before returning to the calling routine. The first call to the subroutine from the main program is with arguments $\left(\mathbf{x}_{0}, 1\right)$.

In order to be concrete, we have to specify what we mean by "calls itself a random number of times". Assume that the coordination number of the lattice is $\mathcal{N}$, so that we can continue a non-reversal walk without self avoidance at every step into $\mathcal{N}-1$ different directions. If we want to obtain a grand canonical ensemble (i.e. a sample in which each configuration is represented in average by const $\times z^{N}$ chains), we have to make in average $P=(\mathcal{N}-1) z$ calls during each call of STEP, and each of the $\mathcal{N}-1$ neighbors must get in average the same number of calls. In incomplete enumeration [11, 9] this is achieved by taking independent probabilities for each possible continuation, i.e. for each free neighbor of $\mathbf{x}$. In our present implementation, in contrast, we chose the neighbors such that the number of continuations shows the smallest fluctuation. More precisely, we first select a random non-reversal direction $\mathbf{e}$ and call STEP at the site $\mathbf{x}^{\prime}=\mathbf{x}+\mathbf{e}$, then we select randomly another of the non-reversal directions 2 . If $P<2$, we select a random number $r \in[0,1]$ and make a second call iff $r<P-1$. Otherwise, we choose a new random direction (different from all previous ones) and continue. If $Q$ is the smallest integer $\leq P$, we try the first $Q$ directions unconditionally, and use $r$ only for the last trial. Obviously, the above assumes that $P \leq \mathcal{N}-1$. If $P=\mathcal{N}-1$, this algorithm is equivalent to exact enumeration.

A conceptually trivial but technically very important generalization consists in letting $P$ depend on $N$. If a sample obtained in this way contains $n_{N, \mathcal{C}}$ chains

\footnotetext{
${ }^{2}$ In not too dense systems it is more efficient to skip the selection of a new direction, and to use the same random $\mathbf{x}^{\prime}$ for all calls.
} 
with configuration $\mathcal{C}$, the contribution of $\mathcal{C}$ to the canonical partition sum is

$$
\frac{n_{N, \mathcal{C}}}{n_{0}(\mathcal{N}-1)^{N}} \prod_{k=1}^{N} P_{N}
$$

As described here, enrichment is much more efficient than incomplete enumeration, typically by factors 5-30 depending on the lattice and on the density of chains. In order to understand why, we have to understand the temporal evolution of the chain length $N$.

As pointed out in [15], $N$ performs essentially a random walk with reflecting boundary at $N=0$. If $z<z_{c}$, there is a bias for $N$ to decrease with (CPU)time, since the average number of returns from subroutine calls is larger than the number of new calls. On the other hand, if $z>z_{c}$, the walk is biased towards an increase of $N$. In either case, the algorithm will be inefficient in creating many statistically independent chains: in the first case since it does not create many chains at all, in the second because most chains will have common first parts and will thus be strongly correlated. The most efficient algorithm is obtained when $z$ is such that there is no net bias. Notice that the latter is true also for dense systems. In this case, there is an additional bias towards smaller chains which increases with $N$ and which is directly proportional to $\rho$. In an efficient algorithm we should thus start with $P_{N} \approx(\mathcal{N}-1) z_{c}$ and increase $P_{N}$ slowly with $N$ in such a way that the net bias remains small.

But absence of bias is not the only criterion for efficiency. The random walk in $N$ is also characterized by an effective diffusion constant $D$. If $D$ is small, then $N$ changes little with time, and the algorithm is obviously inefficient. More precisely, the CPU time needed to create one new chain and erase it again (so that the next independent chain can be built up) is inversely proportional to $D$. For dilute systems one can easily convince oneself that $D=\mathcal{O}(1)$ for incomplete enumeration, while it can be $>>1$ for enrichment [10, 16, 17]. The reason for the latter is easily understood. Assume that $\mathcal{N}>>1$ so that there is very little attrition and we can choose $P_{N}$ close to 1 , more precisely $P_{N}=1+\delta$. In this case most of the steps in the walk will be forward steps, but once we make a backward step (since we selected an occupied site for the next subroutine call) this step will have length $1 / \delta$ in average. Since the net bias is zero, we must thus have $1 / \delta$ forward steps per each back steps, and $D=1 / \delta$. This is of course not rigorous (since the random walk is correlated), but it gives a roughly correct estimate.

The main reason why our algorithms become inefficient at high density (even if $P_{N}$ is chosen optimally, which is already not easy to achieve) is that the above argument break down there, and the effective value of $D$ decreases dramatically. Again this is easily understood heuristically: in a very dense system, we have to make many attempts for each forward step, and the algorithm will spend much time in tempting to prolongate the chain in cases where there simply might be no possible prolongation. Notice that this cannot be avoided by adjusting $P_{N}$ so 
that it would be more appropriate to each specific local configuration. $P_{N}$ has to be chosen uniquely for all local configurations, and has to be such that in average there is no bias. In this sense, polymers in the dense limit are even worse than SAW's in random media where we can chose a different optimized $P_{N}(\mathcal{C})$ for each realization $\mathcal{C}$ of the randomness [9].

In the following, we shall call "tour" the entire random walk between two returns to $N=0$, i.e. the set of all chains generated between two successive returns to the main program. As described above, our algorithm has to be used such that statistics is collected while a tour is going on, but it must be read out only after a tour has been completed. Only in that case eq.(3) is correct. For instance, if we run our algorithm with $P>>(\mathcal{N}-1) z_{c}$ and stop it before finishing a tour, we will "override" some of the repulsive effect of self-avoidance constraint, and the end-to-end distance will in average be smaller than the correct one fi. A crucial question for the following is whether it is possible (and if so, under what conditions) to disregard this problem, and to stop a simulation before a tour is finished. There are situations where this seems more or less the only feasible strategy, since otherwise we either get large finite size corrections (due to short chains which are included near the end points of a tour) or extremely large CPU times.

Thus we would like to follow a strategy where we simulate essentially a single long tour at $z>z_{c}$ which would not be finished at any practically reachable time, and interrupt it after some prescribed number of subroutine calls. It seems that this works indeed surprisingly well in 3 dimensions (though we do not have any analytic proof for it), but it would fail dramatically in $d=2$.

The reason for the failure in $d=2$ is easily understood. There, a typical chain gets stuck in traps as illustrated in fig.1. If we call $A$ the area of the trap, it takes $\sim e^{\left(P-P_{c}\right) A}$ steps (with $\left.P_{c}=(\mathcal{N}-1) z_{c}\right)$ to get out of this trap again. Since the size of the trap can be of the order of the entire lattice, the logarithm of the time needed to get an unbiased sample is thus comparable to the logarithm of the time needed for a complete tour.

In 3- $d$ a chain does not form traps, and we expect this problem to be absent. Indeed, we found numerically that the chain length fluctuates during a tour only little around an average value which is independent of the random number sequence (with a characteristic time of the density autocorrelation function which increases at most as a power of the system size), provided the number of steps is $>V /\left(P-P_{c}\right)$. The latter is the number of steps which would be needed to generate a chain of length $V$ on an infinite lattice. To illustrate the dramatic difference between the 2- $d$ and 3 - $d$ cases, we show in fig.2 histograms of chain lengths reached after $10^{7}$ steps. Each histogram is based on $>100$ independent

\footnotetext{
${ }^{3}$ The effective repulsion results from the fact that a tour which involves larger end-to-end distances will last longer and will thus contribute more to the average distance - provided we follow it until it is finished.
} 
runs. In both cases, $P$ was independent of $N$ and $20 \%$ above the critical value, and the lattices had the same number of sites $\left(512 \times 512\right.$ resp. $\left.64^{3}\right)$. While we see a very wide distribution centered at $N<<V$ for $d=2$ (panel a), the distribution for $d=3$ (panel b) is sharply peaked at a finite density. This density is moreover found to be independent of the lattice size.

The influence of trapping in $d=2$ can also be seen in a different way. In fig. 3 we show the average chain lengths obtained after $m=10^{k}$ steps $(k=3,4,5,6$ and 7$)$ as a function of $z / z_{c}$ (the average maximal length obtained during the first $m$ steps shows very similar behavior, and is only slightly larger, as the chains hardly recede at $z \geq z_{c}$; we used $z_{c}=0.3790524$ [18]). The most conspicuous feature in fig.3 are the pronounced peaks at $z / z_{c}$ slightly above 1 . The steep decay at $z / z_{c} \leq 1$ is of course trivial. Less trivial is the fact that increasing $z$ far above $z_{c}$ does not lead to longer chains. Instead it leads to more trapping. The algorithm becomes more "greedy" in the sense that there is more bias towards an increase of the chain length, and it cannot so easily leave a trap once it has run into it. In fig.3 we show results from lattices of sizes up to $4096 \times 4096$ (which is practically infinite for $10^{7}$ steps). In this limit of infinite lattices, the optimal value of $z / z_{c}$ seems to decrease towards 1 with the number $m$ of steps. This means that greedyness is increasingly punished with chain length. The optimal value of $z-z_{c}$ scales roughly as $1 / \sqrt{m}$, and the chain lengths reached at these optimal values scale as $\sqrt{m}$.

The need for not being too greedy is very reminiscent of optimization problems involving complex functions with many local minima. Prototypes of such problems are provided by the traveling salesman problem, and by the ground state search in spin glasses [19]. The fugacity in the present case is analogous to the inverse temperature when treating such problems by simulated annealing [19]. The main difference is that in our problem we do not have any frozen randomness. In typical hard optimization problems the multitude of false minima is due to randomness in external conditions which remain fixed during the optimization process. In the present case, in contrast, it is the initial stages of the process itself which were random and which remain "frozen" during a finite time which diverges however as the process goes on. In this respect our problem is closer to the complex optimization going on in evolving biological systems [20], where it is also mainly the complexity of the evolutionary process itself (the "co-evolution") which leads to multistability.

\section{Small Densities: Average Monomer Density versus Fugacity}




\subsection{Infinite Volume Limit}

The standard scaling theory for free SAW's [1, 2] describes the behavior of SAW's in the grand canonical ensemble in the limit $z \rightarrow z_{c}-\epsilon$, i.e. if we approach the critical fugacity from the subcritical region. In the present section we propose that this scaling behavior can be extended slightly above $z_{c}$. Thus we should be able to predict also the behavior of dense SAW's in the limit of small densities in terms of the usual critical exponents and scaling functions for non-dense SAW's.

Let us consider the density a finite box of volume $V=L^{d}$ with periodic boundary conditions. Let $\rho(\mathbf{x}, z)$ be the density of monomers in an ensemble where a single polymer is attached with one end to the origin $\mathbf{x}=0$. The length of the monomer is determined implicitly by fixing the fugacity $z$, i.e. by giving each $N$-step chain a weight $\propto z^{N}$. For

$$
\epsilon \equiv \frac{z-z_{c}}{z_{c}}<0
$$

this density becomes independent of $L$ for $L \rightarrow \infty$, and obeys the scaling law [1]

$$
\rho(\mathbf{x}, z) \approx r^{1 / \nu-d} g\left(r^{1 / \nu} \epsilon\right), \quad r=|\mathbf{x}|,
$$

where $g(\zeta)$ is a universal scaling function which can be assumed to be analytic at $\zeta=0$. Notice that the latter is a direct consequence of our choice of scaling variable. More conventional would be the choice $r \epsilon^{\nu}$, but it is clear that this would generate a singularity in $\rho(\mathbf{x}, z)$ at $z=z_{c}$ if $g$ were analytic.

We now assume that eq.(5) holds also for $\epsilon>0$. In this case the polymer should fill a large volume with constant density (except for a region near the origin), and thus $\rho(\mathbf{x}, z) \rightarrow$ const for $\mathbf{x} \rightarrow \infty$. This is only possible if

$$
g(\zeta) \sim \zeta^{d \nu-1},
$$

and from this follows [21]

$$
\rho_{\infty}(z)=\lim _{\mathbf{x} \rightarrow \infty} \rho(\mathbf{x}, z) \sim \epsilon^{d \nu-1} .
$$

In 3 dimensions we had no difficulty in checking this directly. We used systems of different sizes for different values of $z$, but for all $z$ they were large enough so that the fluctuations in chain length were much smaller than the average chain lengths. This is necessary for the average density to be independent of the box size. Otherwise, we would have had a non-negligible chance that the chain length goes occasionally down to zero, which would obviously depend on box sizes. Specifically, this meant for the runs closest to $z_{c}$ that we used box sizes $512^{3}$, and allowed chain lengths up to $3.3 \times 10^{5}$. Notice that this implied also that we had to take statistics without waiting for tours to become completed, as discussed in the last section.

Results from these runs are shown in fig.4. In this plot we used $z_{c}=0.2134908$ which is the value found in [10] (with error \pm 0.0000005 ) for SAW's attached to a 
planar surface. This value is slightly smaller than the previous best value by one of us 22 for free SAW's in infinite volume, but additional unpublished simulations with the latter geometry gave a value in perfect agreement with the above. In contrast, a much larger value with comparable accuracy $(0.2134987 \pm 0.0000010)$ was found from exact enumerations in [23]. We see from fig.4 that we can again exclude the latter. Fig.4 is obviously compatible with scaling. A least square fit would give an exponent $d \nu-1=0.728 \pm 0.1$ or $\nu=0.579 \pm 0.003$, slightly below the best current estimate $\nu=0.587$ [24]. Indeed, a careful look at fig.4 shows a slight downward curvature which might explain this small discrepancy.

Before going on we should point out that eq.(7) has an important consequence for the free energy per monomer, as was pointed out in [3]. If we assume that the free energy is an extensive quantity in the thermodynamic limit, we should have

$$
\frac{1}{N} \ln C_{N, L} \approx-f\left(N / L^{d}\right)
$$

with $f(\rho) \rightarrow-\ln \mu$ for $\rho \rightarrow 0$. If the grand canonical partition sum

$$
Z_{L}(z)=\sum_{N} z^{N} C_{N, L} \approx \int d N z^{N} e^{-N f\left(N / L^{d}\right)}
$$

is estimated by the saddle point approximation, we find the saddle point at

$$
\frac{\partial}{\partial \rho}[\rho f(\rho)]=\ln z
$$

Putting $f(\rho)=-\ln \mu+\Delta f(\rho)=\ln z_{c}+\Delta f(\rho)$, we obtain

$$
-\frac{\partial}{\partial \rho}[\rho \Delta f(\rho)]=\ln \left(z_{c} / z\right) \sim \rho^{1 /(d \nu-1)} .
$$

Integrating this gives [3]

$$
f(\rho)=-\ln \mu+\operatorname{const} \rho^{1 /(d \nu-1)} .
$$

\subsection{Finite Volume Corrections}

In $d=2$ we cannot proceed in the same straightforward way for the reasons explained in sec.2. Instead, we must work on lattices which are small enough, and with fugacities which are close enough to $z_{c}$, so that all tours finish. Indeed, in order not to introduce any bias we have to prescribe the number of tours to be made, not the CPU time.

This means however that we are never really in the limit described by eq.(可), and we have to consider finite- $L$ corrections to it. Assuming that there is a unique diverging length scale $\xi \sim N^{\nu} \sim \epsilon^{-\nu}$ in the polymer problem, any dependence 
on $L$ can only enter through the dimensionless ratio $\xi / L$, and we can make the ansatz

$$
\rho_{L}(z)=\langle N\rangle / L^{d} \approx \epsilon^{d \nu-1} \phi\left(\epsilon L^{1 / \nu}\right)
$$

where $\phi(\zeta)$ is a new scaling function with $\phi(0)$ finite. Notice that this is only an ansatz for the spatially averaged density, and it implies no assumption about its $\mathbf{x}$-dependence.

Alternatively we can start with the canonical ensemble, i.e. with chains of fixed length $N$. Generalizing the usual ansatz for isolated chains in infinite volume and the discussion at the end of the last subsection, we assume

$$
C_{N, L} \approx N^{\gamma-1} e^{-N f\left(N / L^{d}\right)} .
$$

In contrast to eq.(13), the last ansatz should hold also for large densities, and even in the hamiltonian limit $\rho=1$. In the latter, it is known that [3]

$$
C_{N, N} \approx N^{\gamma_{H}-1}\left[\mu_{H}\right]^{N}
$$

where $\gamma_{H}$ depends not only on the dimensionality $d$ but also on the boundary conditions. In $d=2$ it has been conjectured that $\gamma_{H}$ for all lattices is the same as for the Manhattan lattice, where it has the value 35/16 for periodic boundary conditions [3]. In that reference it is also conjectured that all the power behavior in eq. (15) is for $N<L^{2}$ transformed into a term $L^{2\left(\gamma_{H}-1\right)}$. The latter would be in conflict with eq.(14), whence we believe that it is just a trivial mistake.

It is straightforward to show that eqs.(13) and (14) are mutually consistent for any value of $\gamma>0$, provided just that $f(\rho)$ satisfies eq.(12). Indeed, with eq.(12) the ansatz eq. (14) becomes $C_{N, L} \approx N^{\gamma-1} \mu^{N} \exp \left(-\operatorname{const} N \rho^{1 /(d \nu-1)}\right)=$ $N^{\gamma-1} \mu^{N} \chi\left(N^{\nu} / L\right)$. Inserting this into the definition of $\langle N\rangle$ and replacing the summation over $N$ by an integral gives eq. (13).

In order to verify eqs.(14) and (12) in 2 dimensions, we made simulations on square lattices of sizes between $16 \times 16$ and $256 \times 256$. During the simulations $z$ was not kept fixed but depended on $N$ (in order to reach longer chains), but as pointed out in sec. 2 it is trivial to compute $C_{N, L}$ from this. In fig.5 we plotted $\mu(L)=e^{-f(\rho)}$ as defined through eq.(14). Actually, to reduce finite size corrections we replaced the argument of $f$ by $(N-L) / L^{2}$. This is suggested by the fact that the finiteness of the lattice cannot be felt by chains with $N<L$ (we used periodic boundary conditions). We see reasonable scaling, i.e. the functions $f$ defined in this way really depend only on the chain density. We should stress that this would not be so if we had omitted the first factor in eq.(14). To verify eq.(12) we plotted $\Delta f$ in fig.6 against $z-z_{c}$. We see that the prediction $\Delta f \sim \rho^{2}$ is satisfied reasonably well. The agreement is certainly far from perfect, but it improves with increasing $L$, and it would be much worse if we would not have taken into account the first factor in eq.(14). 


\section{Spatial Properties in Dense 2-D Systems}

Some of the most striking predictions concern the spatial structure of dense SAW's in 2 dimensions. In spite of having no obvious control parameter which is set to any special value, the system is predicted to be critical, and several correlation functions should obey non-trivial anomalous scaling laws. We will not attempt here to rederive them but simply refer to [3] for derivations.

\subsection{Grand Canonical End-to-End Distance Distribution}

The first saling law which we want to discuss here concerns the probability that a SAW has an end-to-end distance $R$, irrespective of the number of steps $N$ it involves. Due to the effective repulsion provided by the self avoidance constraint, one should expect that this increases for small $R$. In the spin model analogy this means that the spin-spin correlation increases with distance, a paradoxical result which was put in question in [25].

More precisely, we studied chains starting in the center of a circular disk of radius $r$ cut out from a square lattice. No chain is allowed to reach further than $r$. For $R<<r$, the prediction by Nienhuis [26] is

$$
P(R ; z) \sim R^{3 / 8}
$$

In order to check this, we show in fig.7 results from a disk with radius $r=45$. The fugacity was chosen as $z=0.39$, i.e. about $3 \%$ larger than the critical value. The data are normalized by dividing them by the number of chains with $R=1$ (which is also the number of self avoiding rings). We see in fig.7 large fluctuations at low values of $R$ which are not statistical, but due to the discreteness of the lattice. Statistical errors are much smaller. In the intermediate range of $R$ we indeed observe the predicted scaling regime. The drop at very large $R$ is simple due to the finiteness of the lattice f. $^{\text {t }}$

Analogous data for three dimensions are shown in fig.8. There we show two curves, one for a moderately large spheric volume $(r=40)$ but reasonably large fugacity $\left(z=0.215=1.0071 z_{c}\right)$, the other for a much larger volume $(r=77)$ but much closer to the critical point $\left(z=.214=1.0023 z_{c}\right)$. Both curves show that $P(R ; z)$ increases for small $R$, but the increase is much slower than for $d=2$ and does not seem to follow a power law.

\footnotetext{
${ }^{4}$ the choice of a circular boundary was mainly to keep this drop confined to a narrow region in $R$.
} 


\subsection{Number of Loops vs. Number of Open Chains}

Instead of working at fixed fugacity, let us now consider the number of SAW's and of self avoiding loops at fixed densities. Let us call the latter $C_{N, L}^{(0)}$. We work on square lattices with periodic boundary conditions, and count only loops which pass through the origin. According to [3], we expect

$$
\frac{C_{N, L}^{(0)}}{C_{N, L}} \sim L^{-2 \gamma_{D}}, \quad \gamma_{D}=19 / 16 .
$$

Numerically, this was checked in [3] for $L \leq 16$, at density $N / L^{2}=0.4$. Actually, in general $0.4 L^{2}$ is not integer. Thus for each $L$ the chain length $N$ nearest to $0.4 L^{2}$ had to be taken, and the density slightly fluctuated around 0.4 . While the global behavior was indeed as predicted, it was superimposed by strong odd/even oscillations.

We found that this odd/even effect is mainly due to the following: due to the periodic boundary conditions our lattice is indeed a 2- $d$ torus. Loops can either wind around the torus or have trivial topology (i.e., be homotopic to a point). The latter loops and those which wind twice around the torus always have $N$ even. Walks which wind once around the torus have $N$ even on lattices with even $L$, and vice versa. The total number of of loops depends smoothly on $L$. Thus the number of even- $N$ chains at even $L$ are larger than the number of chains at $L \pm 1$ at any near-by value of $N$. In order to eliminate this effect, we thus plot in fig.9 not precisely the ratio $C_{N, L}^{(0)} / C_{N, L}$ but rather $\left(C_{N-1, L}^{(0)}+2 C_{N, L}^{(0)}+C_{N+1, L}^{(0)}\right) / 2 C_{N, L}$. We see indeed a perfectly straight line on a log-log plot up to $L \approx 40$ (the fluctuations for even larger $L$ are statistical). The theoretical prediction is indicated in fig.9 by a straight line, and is obviously in perfect agreement with our data.

Recently there has been some discussion about the value of $\gamma_{D}$. Based on exact enumerations of collapsed self-attracting SAW's on unbounded lattices, BennettWood et al. 27] found $\gamma_{D}=0.92 \pm 0.09$, while exact solution for the honeycomb lattice with strip geometry gave $\gamma_{D}=1$ [28]. Our data clearly disfavor these values (our estimate is $\gamma_{D}=1.14 \pm 0.05$ ). One reason of the discrepancy with the work of 27 might be that collapsed self-attracting SAW's at finite temperatures are not equivalent to dense SAW's, in contrast to common belief [29]. Another reason might be that $\gamma_{D}$ depends on the boundary condition, again in contrast to common belief.

\subsection{Number of Chains, Absolute Values}

As a last scaling law, we used the same data to check the prediction of [3] that the number of self avoiding chains at fixed density $0<\rho<1$ scales with a $\gamma$-exponent equal to $\gamma_{D}+1$, i.e. as

$$
C_{\rho L^{2}, L} \sim L^{2 \gamma_{D}} e^{-L^{2} \rho f(\rho)}
$$


(compare eq.115). This would imply that the number of loops scales without any power prefactor. It agrees with the behavior in the hamiltonian limit, but we pointed already out in sec.3 that it cannot be correct in the limit $\rho \rightarrow 0$. In the present subsection we shall test whether it holds for intermediate values of $\rho$, more precisely at $\rho=0.4$. To get rid of the unknown constant prefactor in eq.(18), we first form the ratio

$$
C_{N(2 L), 2 L} / C_{N(L), L}
$$

Here $N(L)$ is defined as the integer closest to $0.4 L^{2}$. We then define an effective free energy per monomer as

$$
f_{L}(\rho)=-\frac{\ln \left[C_{N(2 L), 2 L} / C_{N(L), L}\right]}{N(2 L)-N(L)}
$$

and plot $f_{L}(\rho)$ against $1 / L^{2}$. If eq.(18) is correct, this should converge for $L \rightarrow \infty$ towards $f(\rho)$ with slope $\left[\gamma_{D} \ln 4\right] /[3 \rho]$. Otherwise, if eq.(14) is correct, the slope at $1 / L^{2}=0$ should be $[(\gamma-1) \ln 4] /[3 \rho]$. This plot is shown in fig.10. It suggests that eq.(18) is not correct, and eq.(14) is preferred. A more precise statement about the value of $\gamma$ is difficult because of the large fluctuations visible in fig. 10 . They are partly due to the fact that $\rho$ is not strictly constant, but there seems also to be some large scale curvature superimposed. In view of this, our estimate from fig. 10 is $\gamma=1.25 \pm .10$. This could mean that $\gamma$ indeed has its critical value 43/32, and that eq.(14) holds for all finite densities.

\section{$5 \quad$ Very Dense Systems and Hamiltonian Limit}

It is clear from the above that our method becomes less and less efficient if we go to higher and higher densities. Thus it is clear that we can study hamiltonian chains only for very small systems.

In fig. 11 we show results for 2- $d$ (panel a) and 3- $d$ (panel b) systems with free boundary conditions. Due to the boundary conditions, all statistics depend on the starting point of the chains. In fig. 11 we show average values, obtained by averaging over all $L^{d}$ starting points on a square (resp. cubic) lattice. The observable shown is the decadic logarithm of the number of chains. Our results are in good agreement with expectations [3, 7] that

$$
\log \left\langle C_{N, L}\right\rangle=\log \left[L^{-d} \sum_{\text {starting pos. }} C_{N, L}\right] \sim-N f\left(N / L^{d}\right)-B\left(N / L^{d}\right) L^{d-1}
$$

up to logarithmic terms.

The lattice sizes shown in fig.11 are rather modest: $L \leq 10$ for $d=2$, and $L \leq 5$ for $d=3$. Indeed, we tried larger lattices, but we were simply unable to generate even a single hamiltonian chain on any larger lattice with our method. While we estimated that $L=6$ for $d=3$ and $L=11$ for $d=2$ should be feasible with 
unduly large amounts of CPU time, lattices beyond that should be unaccessible with (variants of) our algorithm, and with present day computers.

The data shown in fig.11 were obtained by splitting the range $\left[1, L^{d}-1\right]$ of chain lengths into 3 subintervals $\left[1, N_{1}\right],\left[N_{1}+1, N_{2}\right]$ and $\left[N_{2}+1, L^{d}-1\right]$. On the middle interval we performed the Monte Carlo algorithm as described in sec.2, while we made complete enumerations of all chains in the first and third intervals (typically, $N_{1}=8$ for $d=2$ and $N_{1}=6$ for $N=3$, while $N_{2} \approx 0.75 L^{d}$ ). The exact enumeration on the first interval slightly reduced CPU time and statistical fluctuations, but was not very important for efficiency. In contrast, performing exact enumeration for $N \geq N_{2}$ was crucial. It implied that we found all extensions of random samples of chains with length $N_{2}$.

Indeed, as seen in fig.11, the number of chains decreases dramatically when approaching $N \rightarrow L^{d}$. For $d=2$ and $L=10$, e.g., we see that the number of hamiltonian chains is less than the number of chains with $N \approx 75$ by more than 7 orders of magnitude! If we try to find hamiltonian chains by extending randomly chosen self avoiding chains which fill the lattice partially, we need thus $>10^{7}$ chains of length 75 in order to have just a single success (the data shown in fig. $11 \mathrm{a}$ are based on $6 \times 10^{8}$ chains of length 75 , those of fig. $11 \mathrm{~b}$ - where this factor is $>10^{8}$ - are based on $1.4 \times 10^{9}$ chains).

As seen from fig.11, this decrease of $C_{N, L}$ when approaching the hamiltonian limit becomes rapidly stronger as we increase $L$, rendering our algorithm impracticable on larger lattices. Things are very similar for periodic boundary conditions. The situation is slightly better if we select the chains not randomly but such that dense chains are favored from the very beginning. Thus, the success rate is slightly higher if we use the Rosenbluth method [30], but not enough to treat lattices larger than $11 \times 11$ resp. $6^{3}$.

We should stress that this represents a fundamental obstacle for any algorithm which attempts to generate hamiltonian walks by completing randomly chosen chains which partially fill the lattice. Just such an algorithm was presented in [31]. In that paper first a non-random hamiltonian chain was formed (this is always easy). This chain is cut into two pieces at a randomly chosen site. The shorter half is erased, and completion of the longer part to a new hamiltonian chain is attempted using the Rosenbluth method. If this attempt is successful, the old chain is replaced, while it is restored in the case of failure. After that, a new random cutting site is chosen and the process is repeated. It was claimed in [31] that the algorithm was successful in generating random hamiltonian chains on 2- $d$ lattices with up to $21 \times 21$ sites and with periodic bc. According to our estimates this seems very unlikely. We conjecture that all successful attempts on the largest lattices in [31] involved cutting the chain near one of its ends. The chance for completing it when cutting near the center should have been practically zero, and thus the central piece of the chain should not have been changed at all.

In order to improve the situation, one can use a sample of partial chains which is 
strongly biased in favor of chains which do have at least one hamiltonian extension. We created such samples by growing random chains of length $N \approx L^{d} / 2$ and discarding all chains for which the set of yet unvisited sites is disconnected. On square lattices with $L=11$, e.g., this reduced a sample of chains with $N=60$ by a factor $\approx 10^{3}$. This rendered feasible complete enumeration of all extensions of the remaining chains. Repeating the test for connectivity of the unvisited sites several times improved further the efficiency. In this way we could generate a few hamiltonian chains with $L=12(d=2)$ resp. $L=6(d=3)$, but on larger lattices the method failed again.

Two surprising results were found in [31]: first of all, $\left\langle R_{N}^{2}\right\rangle$ increased faster than linearly with $N,\left\langle R_{N}^{2}\right\rangle \sim N^{1.26}$, and secondly there were strong odd/even oscillations superimposed on that growth. In fig. 12 we show our data for $\left\langle R_{N}^{2}\right\rangle$, also for hamiltonian chains on square lattices with periodic boundary conditions. We see no indication for a faster than linear growth. We do see some odd/even oscillations, but the amplitude is much weaker than that observed in [31], and has opposite sign.

\section{Discussion}

In this paper we have presented simulations of single chains in the dense limit. We have seen that such simulations are very easy in $\geq 3$ dimensions when using a recursive and stochastic implementation of the enrichment method, unless one wants to go to very large densities. More interesting is the 2 - $d$ problem due do universal anomalous scaling laws which should hold there. We were indeed able to test these, with much higher precision than in previous analyses, although our method is much less efficient in $2 d$ than in $3 d$. The reason for the latter is that our algorithm lets SAW's run into 'cages'. In the dense limit we have to use supercritical fugacity, implying that it becomes hard to leave a cage. This becomes the more pronounced the more the fugacity is above its critical value. This is very reminiscent of 'greedy' optimization algorithms in problems with frozen randomness.

In spite of this problem we were able to perform simulations of much longer chains than previously. With one minor exception we verified all scaling laws predicted for dense $2-d$ polymers. This proves that this system is indeed a generic (codimension 1) critical phenomenon.

We also performed simulations of the hamiltonian limit, and showed that neither our algorithm nor any variant thereof can be efficient. If one wants to create a random sample of hamiltonian chains by completing partially filling chains, one has to use for the latter a sample which is strongly biased in favor of such chains which have at least one hamiltonian extension.

In the present paper we have reported only on simulations of a-thermal polymer 
chains. In [16], we have simulated dense polymers near the $\theta$-point. These simulations were essential in eliminating surface effects in the coil-globule transition, and in understanding the nature of this transition. For more details we refer to |16.

Acknowledgement: We are indebted to Drs. Frenkel and Siepmann for discussions about their work. The present work was supported by DFG through SFB 237 and Graduiertenkolleg 'Numerische und Feldtheoretische Methoden in der Hochenergiephysik und Statistischen Mechanik'. 


\section{References}

[1] P.G. de Gennes, Scaling Concepts in Polymer Physics (Cornell Univ. Press, Ithaca, N.Y., 1979)

[2] J. des Cloizeaux and G. Jannink, Polymers in Solution: their Modelling and Structure (Clarendeon Press, Oxford, 1990)

[3] B. Duplantier and H. Saleur, Nucl. Phys. B 290, 291 (1987)

[4] L. Schäfer, Nucl. Phys. B 344, 596 (1990)

[5] J.C. Wheeler and R.G. Petschek, Phys. Rev. B 45, 171 (1992)

[6] P.D. Gujrati, Phys. Rev. A 24, 2096 (1981); B 40, 5140 (1989); Phys. Rev. Lett. 55, 1161 (1985)

[7] B. Duplantier and F. David, J. Stat. Phys. 51, 327 (1988)

[8] P. Bak, Tang and Wiesenfeld, Phys. Rev. Lett. 59, 381 (1987)

[9] P. Grassberger, J. Phys. A 26, 1023 (1993)

[10] P. Grassberger and R. Hegger, J. Phys. A 27, 4069 (1994)

[11] S. Redner and P.J. Reynolds, J. Phys. A 14, 2679 (1981)

[12] F.T. Wall and J.J. Erpenbeck, J. Chem. Phys. 30, 634, 637 (1959)

[13] G.F. Tuthill, J. Chem. Phys. 92, 3179 (1990)

[14] G.F. Tuthill and D.J. Glover, J. Chem. Phys. 94, 8408 (1991)

[15] A. Beretti and A.D. Sokal, J. Stat. Phys. 40, 483 (1985)

[16] P. Grassberger and R. Hegger, preprint submitted to J. Chem. Phys. (1994)

[17] P. Grassberger, R. Hegger and L. Schäfer, preprint (1994)

[18] A.J. Guttmann and I.G. Enting, J. Phys. A 21, L165 (1988)

[19] M. Mézard, G. Parisi and M.A. Virasoro, Spin Glasses Theory and Beyond (World Scientific, Singapore 1988)

[20] S. Kauffman, Requirements for Evolvability in Complex Systems, in Complexity, Entropy and the Physics of Information, ed. W.H. Zurek (AddisonWesley, Redwood City 1990)

[21] H. Saleur, Phys. Rev. B 35, 3657 (1987)

[22] P. Grassberger, J. Phys. 26, 2769 (1993) 
[23] D. MacDonald, D.L. Hunter, K.Kelly and N. Jan, J. Phys. A 25, 1429 (1992)

[24] B.G. Nickel, Macromolecules 24, 1358 (1991)

[25] P.D. Gujrati, Phys. Rev. B 31, 4375 (1981)

[26] B. Nienhuis, J. Stat. Phys. 34, 731 (1984)

[27] D. Bennett-Wood, R. Brak, A.J. Guttmann, A.L. Owczarek and T. Prellberg, J. Phys. A 27, L1 (1994)

[28] M.T. Batchelor, J. Suzuki and C.M. Yung, Canberra preprint (1994)

[29] A.L. Owczarek, T. Prellberg and R. Brak, Phys. Rev. Lett. 71, 4275 (1993)

[30] M.N. Rosenbluth and A.W. Rosenbluth, J. Chem. Phys. 23, 356 (1955)

[31] J.I. Siepmann and D. Frenkel, Mol. Phys. 75, 59 (1992) 


\section{Figure Captions:}

Fig.1: Trapped 2-d SAW. In supercritical simulations using the present algorithm, there is a bias towards increase of the chain length, and it can take extremely long until such a trap is left again.

Fig.2: Histograms of chain lengths reached after $10^{7}$ subroutine calls in supercritical simulations. Panel a: 2 - $d$ lattice of $512 \times 512$ sites, $\mu=0.454863$; panel b: 3 - $d$ lattice of size $64^{3}, \mu=0.256189$.

Fig.3: Average chain lengths obtained after $10^{k}$ subroutine calls $(k=3, \ldots 7)$ on 2 - $d$ lattices of sizes up to $4096 \times 4096$, plotted against $z / z_{c}$. For each value of $z$, data are averages over 500 independent runs.

Fig.4: Log-log plot of the density of supercritical SAW's filling a finite but large $3-d$ volume, in dependence of the fugacity $\mu$. The critical fugacity was chosen according to [10].

Fig.5: Effective coordination numbers per monomer for 2- $d$ chains, after dividing out the logarithmic corrections from $C_{N, L}$ (see eq.(14). On the abscissa is plotted the corrected density $(N-L) / L^{d}$.

Fig.6: Log-log plot of $\Delta f$ against the corrected density, obtained from the same data as in fig.5.

Fig.7: Log-log plot of the number of chains with end-to-end distance $R$ plotted against $R^{2}$. These data were obtained on a disk of radius $r=45$ at fixed fugacity $z=0.39$. They are normalized to 1 at $R=1$. The straight line has slope $3 / 16$, and indicates the theoretical prediction.

Fig.8: Simular to fig.7 but for three dimensions.

Fig.9: Number of loops divided by number of chains, both at fixed density $\rho=$ 0.4 , plotted against $L$. The theoretical prediction is indicated by the straight line with slope $-19 / 8$.

Fig.10: Free energy per monomer $f_{L}(\rho=0.4)$ against $1 / L^{2}$. Indeed, since $0.4 L^{2}$ is generally not an integer, the density slightly oscillates around the value 0.4 . This is mainly responsible for the fluctuations seen in the data, as statistical errors are comparable to the size of the dots.

Fig.11: Decadic logarithms of the number of chains on lattices with free boundaries, averaged over the position of the starting point. For $d=2$ (panel a), curves are for $L=4, \ldots 10$, while they correspond to $L=3,4$ and 5 for $d=3$ (panel b).

Fig.12: $\left\langle R^{2}\right\rangle^{1 / 2}$ against $L$ for hamiltonian chains on square lattices with periodic boundary conditions. The data for $L \leq 6$ are based on exact enumerations and thus free of statistical errors. 